\section{Buchrezension zu: Sich besser präsentieren}

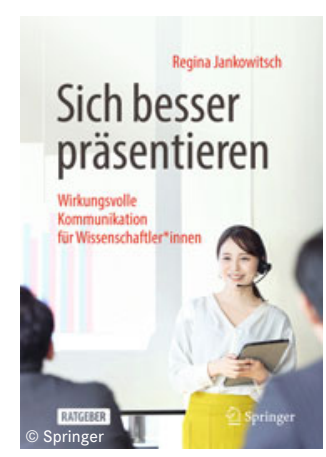

Sich besser präsentieren Wirkungsvolle Kommunikation für Wissenschaftler*innen Regina Maria Jankowitsch

209 S., Springer, 2021, SC, 27,99 €. ISBN 978-3658331740

Auch als E-Book erhältlich

DOI: $10.1007 / \mathrm{s} 12268-021-1657-6$ (c) Der Autor 2021

Wenn sich die Zuhörer:innen langweilen, liegt es nicht an den Zuhörer:innen. Diese banale und manchmal brutale - Wahrheit ist leider noch nicht in allen Köpfen angekommen, sonst bestünde kein Bedarf an der inzwischen mannigfaltigen Literatur zum Thema (Wissenschafts-)Kommunikation. Auch Regina Maria Jankowitsch, Coachin, Autorin und Moderatorin, nimmt die challenge an und entwickelt ihre mission auf 209 Seiten. Diese besteht darin, Wissenschaftler:innen mit steter Wiederholung gelungene Kommunikation nahe zu bringen.

Der Vorteil dieses Buchs ist, dass man es als Büfett nutzen und sich das herauspicken kann, was gerade interessiert; der Nachteil ist, dass das Büfett wenig abwechslungsreich ist, die Garnierung wechselt, die Rezepte jedoch immer die gleichen sind um eines der Bilder zu nutzen, die die Autorin empfiehlt. Was, um die nicht so überzeugenden Dinge gleich zu Beginn abzuhandeln, ebenfalls nicht richtig gelungen ist: die Zielgruppendefinition. On paper richtet sich das Buch an Professor:innen, Lehrende und auch Journalist:innen, Studieren- de und interessierte Laien, in fact ist es für Laien eher mäßig interessant, für Journalist:innen, die ihr Handwerk beherrschen, redundant, für Studierende, die seltenst Drittmittel einwerben und Berufungskommissionen überzeugen wollen, zu 50 Prozent nicht relevant. Empfohlen werden kann es für alle, die hauptberuflich „in Wissenschaft machen“, vom Prae-Doc-Status bis zur Professur inklusive wissenschaftsunterstützendem Bereich, und für diejenigen, die sich bisher kaum mit Kommunikation beschäftigt haben.

Inhaltlich wendet Regina Maria Jankowitsch die bekannte AIDARegel (Attention, Interest, Desire, Action) auf die Darstellung von Wissenschaft und ihrer Erkenntnisse sowie dem Selbstmarketing von Wissenschaftler:innen an. Dadurch wiederholen sich die Anleitungen und Checklisten zwangsläufig, was kein Manko ist, denn vermutlich wird das Buch im Sinne des Büfetts genutzt. Dafür ist es gut strukturiert, von den Basics („Spielregeln professioneller Kommunikation") geht es über die Drittmittel- und Berufungskommunikation über die Kommunikation auf der Bühne (Vortrag und Diskussionsveranstaltung) bis zur Kommunikation mit der Presse und im digitalen Bereich. Dieses Kapitel („Digital Audio und Podcast") wirkt ein wenig gewollt und sub Corona ergänzt, wie insgesamt die digitale Kommunikation für den Geschmack dieses Lesers eine Spur mehr Aufmerksamkeit verdient hätte.

Zuletzt zur Sprache und zum Stil. Regina Maria Jankowitsch geht mit gutem Beispiel voran und stellt ihr Licht in amerikanischem Stil nicht unter den Scheffel. Gleich zu Beginn erfährt man, wie groß ihre Expertise ist und wen sie zur Mitarbeit gewinnen konnte; das kann man gut finden, daran kann man sich stoßen, das kann man als Unsicherheit interpretieren. Ähnlich verhält es sich mit den Wiederholungen, wenn man das Buch in Gänze liest, und den hervorgehobenen „Tipps“, die, je nach Lesart, als Funktionsbefehle interpretiert werden können. Den Lesefluss stören die Inkohärenz beim Gendern, die wechselnden Formate, der Flattersatz und die befehlsartige Nutzung von Worten in Großbuchstaben. Doch das sind in der Gesamtschau Petitessen, die die Empfehlung nicht schmälern sollen.

Jochen O. Ley,

Humboldt-Universität zu Berlin, jochen.ley@uv.hu-berlin.de

Diese Rezension erscheint Open Access. *

\footnotetext{
* Funding note: Open Access funding enabled and organized by Projekt DEAL. Open Access: Dieser Artikel wird unter der Creative Commons Namensnennung 4.0 International Lizenz veröffentlicht, welche die Nutzung, Vervielfältigung, Bearbeitung, Verbreitung und Wiedergabe in jeglichem Medium und Format erlaubt, sofern Sie den/die ursprünglichen Autor(en) und die Quelle ordnungsgemäß nennen, einen Link zur Creative Commons Lizenz beifügen und angeben, ob Änderungen vorgenommen wurden. Die in diesem Artikel enthaltenen Bilder und sonstiges Drittmaterial unterliegen ebenfalls der genannten Creative Commons Lizenz, sofern sich aus der Abbildungslegende nichts anderes ergibt. Sofern das betreffende Material nicht unter der genannten Creative Commons Lizenz steht und die betreffende Handlung nicht nach gesetzlichen Vorschriften erlaubt ist, ist für die oben aufgeführten Weiterverwendungen des Materials die Einwilligung des jeweiligen tails zur Lizenz entnehmen Sie bitte der Lizenzinformation auf http://creativecommons.org/licenses/ by $/ 4.0 /$ deed.de.
} 